\title{
Prospective Biomarker Trials Chemo NO and NNBC-3 Europe Validate the Clinical Utility of Invasion Markers UPA and PAI-1 in Node-Negative Breast Cancer
}

\author{
Nadia Harbeck ${ }^{a}$ Manfred Schmitt ${ }^{a} \quad$ Martina Vetter $^{b} \quad$ Janna Krol $^{\mathrm{a}} \quad$ Daniela Paepke $^{\mathrm{a}}$ \\ Mathias Uhlig ${ }^{\mathrm{c}}$ Stefan Paepke ${ }^{\mathrm{a}}$ Fritz Jänicke ${ }^{\mathrm{d}}$ Anneke Geurts-Moespot ${ }^{\mathrm{e}}$ \\ Gunter von Minckwitz ${ }^{\mathrm{c}}$ Fred Sweep ${ }^{\mathrm{C}}$ Christoph Thomssen ${ }^{\mathrm{b}}$ \\ a Frauenklinik und Poliklinik der Technischen Universität München, \\ ${ }^{b}$ Frauenklinik, Martin-Luther-Universität Halle, \\ c German Breast Group, Neu-Isenburg, \\ dUniversitätsfrauenklinik Eppendorf, Hamburg, Germany \\ e Department of Chemical Endocrinology, Radboud University Nijmegen Medical Centre, The Netherlands
}

\section{Introduction}

Urokinase-type plasminogen activator (uPA) and its inhibitor plasminogen activator inhibitor-1 (PAI-1) are key factors in tumour invasion and metastasis. Increased levels of UPA and/or PAI-1 in primary tumour tissues correlate with tumour aggressiveness and poor patient outcome $[1,2]$. In primary breast cancer, Duffy et al. [3] in 1988 were the first to show that high enzymatic activity of uPA in primary breast cancer tissues was correlated with advanced tumour stage and poor clinical outcome. In 1989, Jänicke et al. [4] demonstrated that high uPA antigen levels in primary tumour tissue measured by enzyme-linked immunosorbent assay (ELISA) also predicted poor prognosis. At the same time, it also became apparent that not only antigen levels of uPA but also those of PAI-1 are of prognostic importance in node-negative and node-positive breast cancer patients $[4,5]$. Subsequently, many international, mostly European, researchers validated that primary breast cancer patients, and in particular node-negative patients with high tumour tissue antigen content of uPA and/or PAI-1, have a worse probability of disease-free survival (DFS) and overall survival (OS) than patients with low levels of either or both biomarkers [6-9]. For clinical utility, the combination of UPA/PAI-1 (both low versus either or both high) is superior to either factor taken alone regarding risk group assessment [10]. uPA and PAI-1 render prognostic information independent of established prognostic factors such as tumour size, tumour grade, steroid hormone receptor status, menopausal status [10] and even HER2 status [11-13]

Next to their prognostic impact, uPA/PAI-1 also provide predictive information in early breast cancer. Patients with high uPA/PAI-1 have an enhanced benefit from adjuvant chemotherapy compared to those with low levels. Regarding adjuvant endocrine therapy, no such interaction between benefit and uPA/PAI-1 levels could be found [14]. A large meta-analysis conducted by the European Organisation for Research and Treatment of Cancer (EORTC) Receptor and Biomarker Group, comprising 8377 breast cancer patients from 18 independent collectives, validated the prognostic [15] and predictive impact of uPA/PAI-1 [16], thus achieving the highest level of evidence
(LOE-I) for clinical utility of a cancer-associated biomarker, according to the American Society of Clinical Oncology (ASCO) tumour marker utility grading system (TMUGS) [17]

\section{Chemo NO Trial}

The Chemo N0 trial is a prospective randomised multicentre breast cancer therapy trial in which 689 node-negative primary breast cancer patients were enrolled in 14 study centres in Germany and Slovenia between 1993 and 1998 [18]. Principal investigator was Prof. Dr. Fritz Jänicke, formerly at the Technical University of Munich, who is now at the University of Hamburg. In this trial (fig. 1), uPA and PAI-1 antigen levels were determined in primary tumour tissue extracts by commercially available ELISA kits (American Diagnostica Inc., Stamford, CT, USA) and used for patient stratification: Patients with low uPA and PAI-1 levels were only observed; patients with high uPA and/or PAI-1 were randomised to either adjuvant chemotherapy with 6 cycles of cyclophosphamide/methotrexate/5-fluorouracil (CMF) or observation only. The prognostic impact of UPA/PAI-1 was then evaluated prospectively by comparing the two observation arms (low versus high uPA/PAI-1), whereas comparison of the two randomised arms allowed prospective evaluation of the predictive benefit of uPA/PAI-1 regarding benefit of adjuvant chemotherapy (fig. 1).

The first scheduled interim analysis of the Chemo N0 trial after a median follow-up time of 32 months $(n=556)$ validated prospectively the statistically independent prognostic impact of uPA/PAI-1 regarding DFS [18]. In addition, previously optimised cut-off values for uPA and PAI-1 to discriminate between low and high uPA/PAI-1 were confirmed. The second analysis after a median follow-up time of 50 months comprising 647 patients substantiated the prognostic impact of uPA/PAI-1 regarding DFS as well as OS. Of the 647 patients, 283 had low uPA/PAI-1 and 364 had high uPA and/or PAI-1. The actuarial 3-year recurrence rate for patients with low uPA/PAI-1 was $6.3 \%$, but was $14.2 \%(p=0.009)$ for those with high uPA/PAI-1 in the observation group. Thus, this second interim analysis revealed that node-negative breast cancer patients with low uPA/PAI-1 have an es-

\section{KARGER}

Fax +497614520714

Information@Karger.de

www.karger.com (c) 2008 S. Karger GmbH, Freiburg

Accessible online at:

www.karger.com/brc
Prof. Dr. med. Nadia Harbeck

Frauenklinik der Technischen Universität München

Ismaninger Str. 22, 81675 München, Germany

Tel. +49 89 4140-4596, Fax -4846

nadia.harbeck@1rz.tum.de 


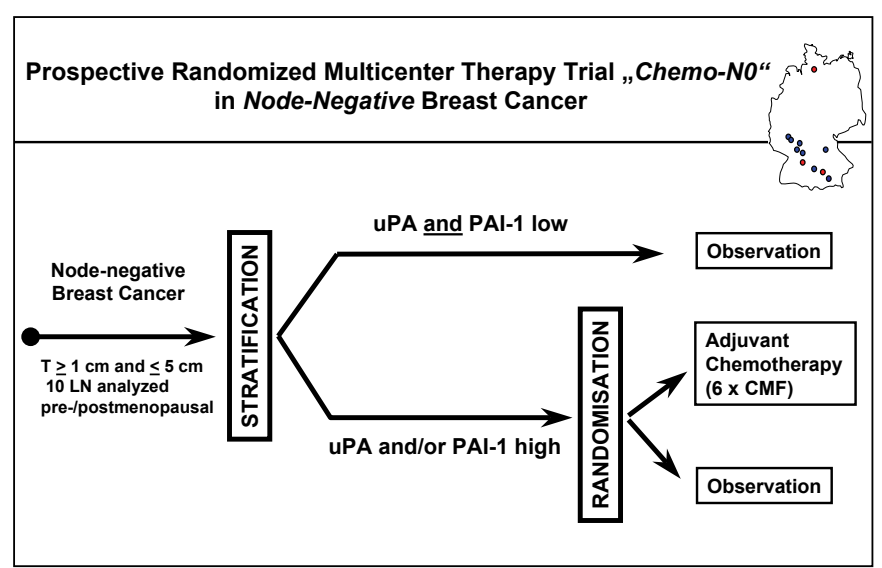

Fig. 1. Chemo N0 trial: Study design [18]. Node-negative breast cancer patients were stratified according to the uPA/PAI-1 levels in their primary tumour tissue: Patients with low levels were just observed; those with high levels were randomised for $\mathrm{CMF}$ chemotherapy or observation only.

timated 5-year OS rate of $\sim 95 \%$ even in the absence of any adjuvant systemic therapy and can thus be considered as low-risk [19].

In the Chemo N0 trial, node-negative patients with high uPA/PAI-1 were either randomised to CMF-based chemotherapy or to observation only, thereby evaluating the clinical benefit of a therapeutic intervention in high-uPA/PAI-1 patients. Even after a short follow-up period of 32 months, a considerable benefit from adjuvant CMF chemotherapy was observed in high-uPA/PAI-1 node-negative breast cancer patients [18]. This therapy benefit was still valid after a longer median follow-up time of 50 months [19]. High-risk patients in the chemotherapy group had a $36.9 \%$ lower estimated probability of disease recurrence than high-risk patients in the observation group (intention-to-treat analysis relative risk (RR) $0.63 ; 95 \%$ confidence interval $(\mathrm{CI}) 0.33-1.20 ; \mathrm{p}=0.16)$. In a per-protocol analysis, the treatment benefit reached statistical significance (RR $0.42 ; 95 \% \mathrm{CI}$ $0.20-0.87 ; \mathrm{p}=0.019$ ) for DFS and persisted with regard to OS. The prospective randomised multicentre clinical Chemo N0 trial therefore not only validated the prognostic value of UPA/PAI-1 in node-negative breast cancer patients, but also demonstrated their predictive impact at the highest level of evidence, LOE-I.

The final 10-year follow-up of the Chemo N0 trial is currently being finalised. Based on the long-term observation within the trial, a first comparison between actual 10-year patient outcome and Adjuvant Online 10-year survival estimates (www.adjuvantonline.com) was possible. Preliminary results indicate that uPA/PAI-1 testing may enhance outcome estimates by the epidemiological data-driven webbased algorithm Adjuvant Online [20]. Whereas for the whole Chemo N0 patient collective, Adjuvant Online 10-year survival estimates seem rather accurate, Adjuvant Online tends to overestimate risk in those patients considered as low-risk according to uPA/PAI-1 and to underestimate risk in those patients classified as high-risk by uPA/PAI-1.

In conclusion, the Chemo N0 breast cancer trial was able to answer a number of clinical questions but left others still open. Determination of UPA and PAI-1 in primary tumour tissue extracts was possible in a multicentre setting ( $\mathrm{n}=6$ participating laboratories) for clinical decision-making. Node-negative breast cancer patients with low uPA and PAI-1, which account for about half of the node-negative breast cancer patients, have a rather low risk of disease recurrence and can therefore be spared the burden of adjuvant systemic chemotherapy. However, optimal chemotherapy for high-risk patients according to their high uPA and/or PAI-1 levels, who are at risk to develop metastasis, still needs to be determined.
NNBC3-recruitment at 01.07.2008 $(\mathrm{N}=3136)$

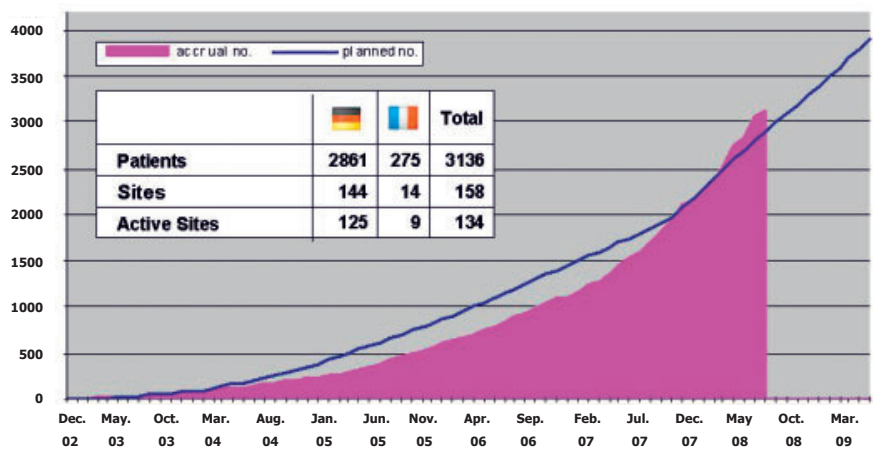

Fig. 2. Current NNBC-3 Europe recruitment status. On July 1, 2008, 3136 patients had been recruited by 134 clinical centres in Germany and France.

\section{NNBC-3 Europe Trial}

Following the results of the Chemo N0 trial, a second prospective multicentre phase III therapy trial, the Node-Negative Breast Cancer3 (NNBC-3) Europe trial, was launched in 2003 and it is still open for patient recruitment. Principal investigators are Prof. Dr. Christoph Thomssen ('Leiter der Klinischen Prüfung' according to German law), University of Halle, and Prof. Dr. Nadia Harbeck, Technical University of Munich, Germany.

The NNBC-3 Europe trial has two primary objectives: (1) to compare risk assessment and clinical outcome based on the tumour-biological factors UPA/PAI-1 to those based on established, clinical and pathomorphological factors and (2) to optimise adjuvant chemotherapy for high-risk node-negative breast cancer patients. The NNBC-3 Europe trial is an Intergroup effort conducted in co-operation with the German 'Arbeitsgemeinschaft Gynäkologische Onkologie' (AGO), the EORTC PathoBiology Group, and the German Breast Group. At present (July 2008), recruitment is at 3136 patients coming from 134 clinical centres in Germany and France (fig. 2). Main eligibility criteria include node-negative primary breast cancer, age 18-70 years, and a tumour size between 0.5 and $5 \mathrm{~cm}$. Centres are asked to select their preferred way of risk group assessment criteria (established clinico-pathological criteria or using uPA/PAI-1 levels in primary tumour tissue extracts) upfront for the duration of the trial (fig. 3). Those centres opting for risk assessment by established factors use an algorithm based on the St. Gallen 2005 consensus meeting recommendations [21]. Using these criteria, a node-negative breast cancer patient fulfilling any of the following criteria is considered as highrisk regarding disease recurrence: (1) age $<35$ years, peritumoural vascular invasion, tumour grade 3 , progesterone receptor $(\mathrm{PgR})-/$ estrogen receptor (ER)+ or PgR-/ER-, or HER2 positive, or (2) tumour grade $\mathrm{G} 2$ and tumour size $>2 \mathrm{~cm}$. The second risk assessment option applies the levels of the invasion markers uPA and PAI-1 as determined in primary tumour tissue extracts and uses the same cut-off values as had been used in the Chemo N0 protocol: $3 \mathrm{ng} / \mathrm{mg}$ protein for $\mathrm{uPA}$ and $14 \mathrm{ng} / \mathrm{mg}$ protein for PAI-1 [18].

For all patients, tumour grade and age are decisive for risk assessment (fig. 3), with all patients younger than 35 years of age and those with G3 tumours considered as high-risk and thus randomised for the chemotherapy option. Patients with G1 tumours are considered as low-risk and allocated to the observation arm. For patients with G2 tumours, further risk stratification according to the St. Gallen-based algorithm as outlined above or according to uPA/PAI-1 criteria is performed. Patients with low UPA/PAI-1 are considered as low-risk and thus allocated to the observation arm. Patients with high uPA and/or 
A

Fig. 3. NNBC-3 Europe study design: Clinical centres can opt for either established clinicopathological criteria $(\mathbf{A})$ or uPA/PAI-1 levels in primary tumour tissue extracts $(\mathbf{B})$ as their criteria for risk group assessment. For all patients, tumour grade and age are decisive for risk assessment.

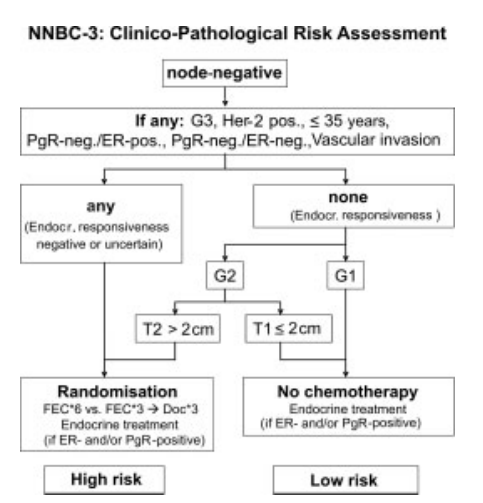

B

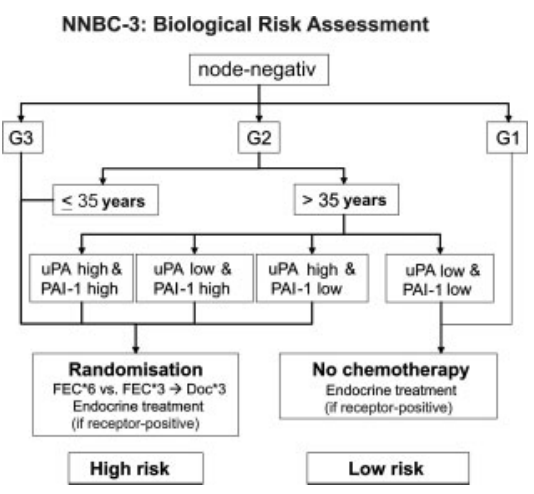

PAI-1 are considered as high-risk and thus randomised to one of the two chemotherapy regimens.

In the NNBC-3 Europe trial, all patients defined as being at high risk for disease recurrence are randomised to either 6 cycles of 5-fluorouracil/epirubicine/cyclophosphamide $\left(\mathrm{FE}_{100} \mathrm{C}\right) 3$-weekly or to $3 \mathrm{cy}$ cles of $\mathrm{FE}_{100} \mathrm{C}$ followed by 3 cycles of docetaxel 3-weekly, as used in the PACS 001 trial [22]. All patients in the high-risk and in the lowrisk group, provided that their tumours are steroid hormone receptor positive, receive endocrine therapy according to the current AGO guidelines [23]. According to these guidelines [23], radiation therapy is also permitted. For HER2-positive patients, 1 year of adjuvant trastuzumab therapy is recommended.

Regarding risk-grouping, so far 2038 patients have been assessed using tumour-biological criteria and $38 \%$ of these patients have been considered as low-risk and $62 \%$ as high-risk (fig. 4A). Of the 1111 patients assessed for their relapse risk using clinico-pathological criteria, $31 \%$ have been considered as low-risk and $69 \%$ as high-risk (fig. 4B). With the group of G2 tumours, using uPA/PAI- $142 \%$ of the patients have been considered as low-risk, and $43 \%$ using clinico-pathological criteria (fig. 5). These low-risk patients have then been spared adjuvant chemotherapy within the trial setting.

In summary, the overall goals of the NNBC-3 Europe trial are to compare both risk stratification methods - the clinico-pathological and the tumour-biological one - and to determine the benefit of a sequential anthracycline-docetaxel regimen in high-risk node-negative breast cancer. Patient recruitment has been rapid after initial delays (most likely due to establishment of logistics for uPA/PAI-1 determination, e.g. tumour tissue freezing, transport, time lines for result reporting etc. within the centres) so that the NNBC-3 Europe trial is expected to complete recruitment in early 2009.

\section{Quality Assurance of the uPA/PAI-1 Test within the Chemo NO and NNBC-3 Europe Trials}

High levels of antigen uPA and/or PAI-1 in cytosolic extracts of human primary breast cancer tissue have been associated with rapid disease progression and shorter OS [19]. The conclusions are drawn from results obtained from different types of UPA and PAI-1 ELISAs, employing different antibodies and different standards. Notwithstanding the differences in analytical features, the prognostic impact of UPA and PAI- 1 in primary breast cancer has been substantiated at the highest level of evidence as outlined above. In the present prospective multicentre clinical trial (NNBC-3 Europe), 134 centres are participating, whereas assessment of UPA and PAI-1 concentrations in tissue extracts is performed in a limited number of core laboratories all using the same commercially available assay (FEMTELLE ${ }^{\circledR}$ kit; American Diagnostica, Stamford, CT, USA).
Once a week, the collected frozen tissues are sent overnight from the participating hospital to one of the core laboratories. All these laboratories use standardised protocols for the preparation of cytosolic extracts and for the measurement of UPA, PAI-1, and total protein. Moreover, their participation in an external quality assurance (EQA) programme developed by the central quality assurance (QA) laboratory (Dept. of Chemical Endocrinology, Radboud University Nijmegen Medical Centre) is mandatory.

Before starting measurements within the NNBC-3 Europe trial, the performance of all laboratories was carefully screened in a pilot test study which included monitoring of within-laboratory, within-run and within-laboratory between-run variations using lyophilised cytosolic materials provided by the central EQA laboratory. Each participant received 9 samples for UPA and PAI-1 (3 sets of 3 vials which were supposed to be analysed in 3 separate assay runs with at least 2-week intervals using the FEMTELLE kit). Also included were 30 quality control (QC) samples for the measurement of total protein of the tissue extract using the Pierce $\mathrm{BCA}^{\mathrm{TM}}$ protein assay kit. Each set of vials within a run contains the same samples, although the sequence differs from run to run. This enables the EQA laboratory to calculate between-run and between-laboratory coefficients of variation (CVs). All laboratories have to achieve within-laboratory within-run and within-laboratory between-run CVs of less than 10 and $15 \%$, respectively. Only then will the central EQA laboratory and the NNBC-3 Europe study coordinator (Prof. Dr. Christoph Thomssen) give the participating laboratory a green light to start with uPA, PAI-1 and total protein measurements for other institutes within this clinical trial. During the entire study period, participants include in each of their UPA and PAI-1 assay runs a lyophilised external reference vial prepared from xenograft tissue (recovered from nude mice implanted with the breast cancer cell line MDA-MB-231 [24]) and an internal control in order to monitor within-lab between-run variations. The reference preparation is checked with regard to homogeneity, storage stability, and parallelism before it is used in the EQA trials. The target values of UPA and PAI-1 were established during long-term measurements using the FEMTELLE kit in 5 different institutes. Monitoring these target values enables checking for analytical drift and shift in absolute analyte values during measurements over time.

Every 2 months (i.e. 6 times a year), a set of 3 EQA samples are analysed for UPA and PAI- 1 in one assay run, as well as 3 samples for protein determination. The composition of the sets differs from set to set. The use of identical vials in one set, different dilutions in one set and/or identical vials over the sets enables the EQA laboratory to monitor long-term performance and within- and between-assay CV (altogether 5 different concentrations for UPA and PAI-1 and 9 for the total protein determination). Each year, all QA data are presented and discussed at the NNBC-3 Europe investigators meeting by a staff member of the Nijmegen laboratory. This central EQA laboratory an- 


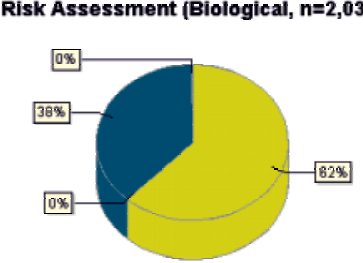

HIGH HIGH \{G2 Rule)
Risk Assessment (Clin-Patho, $n=1,111$ )

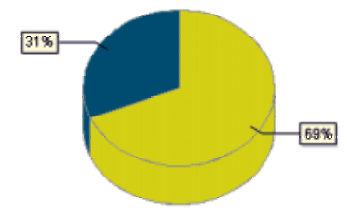

HIIGH LOW

Fig. 4. NNBC-3 Europe trial - actual risk assessment: Using tumourbiological criteria, 2038 patients were assessed and $38 \%$ of these patients were considered as low-risk and $62 \%$ as high-risk (A). Using clinicopathological criteria, 1111 patients were assessed and $31 \%$ were considered as low-risk and 69\% as high-risk (B).

nually provides each laboratory with a report on their intra-laboratory and inter-laboratory CVs, together with a note of the overall betweenlaboratory CVs of all participants. If a particular laboratory displays a substantial shift in analytical performance, the central EQA laboratory contacts this laboratory and the whole process within the laboratory is scrutinised in order to detect the potential source of error.

Up to now, the within-laboratory within-assay CVs for uPA are approximately $4 \%$, and $5 \%$ for PAI- 1 . Obviously, the within-laboratory between-assay CVs are higher. During the first QA round, most laboratories had CVs below $15 \%$ but some had CVs higher than $25 \%$. In the second year, all laboratories scored acceptable within-lab betweenrun CVs for uPA and PAI-1 below 13\%. As far as the protein assay is concerned, the within-lab within-assay, the within-lab between-assay, and the between-lab CVs amount to 5,7 and 14\%, respectively.

In summary, a total of 10 laboratories participated in the pilot test study for the NNBC-3 Europe study; they received standardised protocols for the uPA, PAI-1, and protein assays, as well as lyophilised external quality control (EQC) samples. For 7 out of 10 laboratories, there was no reason to question their analytical expertise, whereas in 3 laboratories the performance did not meet the strict criteria set beforehand and these laboratories received a new set of samples and new instructions. After re-analyses, these laboratories also met the criteria. All participating laboratories are able to measure uPA and PAI-1 with an acceptable precision. However, the within-laboratory between-run and consequently the between-lab CVs are less reproducible; in particular during the first year the PAI-1 data were moderate. The uPA and PAI-1 assay results during the second year are much more consistent than those of the first trial year.

A

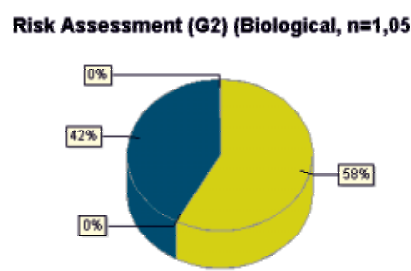

EHIGH HIGH (O2 Rule) 1 LOW 1 LOW (G2 Rule)

Fig. 5. NNBC-3 Europe trial - actual risk assessment for patients with G2 tumours. In the group of patients with G2 tumours, using uPA/PAI-1, $42 \%$ of the patients were considered as low-risk (A), and $43 \%$ using clinico-pathological criteria (B).
To monitor within-laboratory between-assay CVs, and between-laboratory CVs, use of control preparations is of utmost importance. Clearly, a strict uniform way of sample processing and handling within the laboratories is essential for overall quality of assay results. Participation in an EQA program is a prerequisite in multicentre studies [25].

\section{Conclusions}

The invasion markers uPA and PAI-1 have been validated at the highest level of evidence regarding their prognostic and predictive impact in primary breast cancer. The Chemo N0 trial as well as the ongoing NNBC-3 Europe trial clearly demonstrate the feasibility of uPA/PAI-1 testing under clinical routine in a multicentre setting with good quality assurance. Comparison of clinical and tumour-biological data from NNBC-3 Europe with the other two international trials in node-negative breast cancer (MINDACT and TailorX) would be ideal in order to achieve optimal risk group stratification in node-negative (and possibly node-positive) breast cancer.

Given the validated clinical relevance of uPA/PAI-1 and the standardised ELISA assay system suitable for multicentre testing, the German Working Group for Gynaecological Oncology (AGO) guidelines [23] as well as the ASCO guidelines [26] recommend both biomarkers for risk group classification and routine clinical decisionmaking in node-negative breast cancer, next to established clinicopathological factors.

\section{References}

1 Andreasen PA, Kjoller L, Christensen L, Duffy MJ: The urokinase-type plasminogen activator system in cancer metastasis: a review. Int J Cancer 1997;72:1-22.

2 Schmitt M, Wilhelm O, Reuning U, et al.: The plasminogen activation system as a novel target for therapeutic strategies. Fibrinolysis 2000;14:114 132.

-3 Duffy MJ, O’Grady P, Devaney D, O'Siorain L, Fennelly JJ, Lijnen HJ: Urokinase-plasminogen activator, a marker for aggressive breast carcinomas. Preliminary report. Cancer 1988;62:531-533.

4 Jänicke F, Schmitt M, Ulm K, Gossner W, Graeff J: Urokinase-type plasminogen activator antigen and early relapse in breast cancer. Lancet 1989;2 1049
5 Jänicke F, Schmitt M, Graeff H: Clinical relevance of the urokinase-type and tissue-type plasminogen activators and of their type 1 inhibitor in breast cancer. Semin Thromb Hemost 1991;17:303-312.

6 Kim SJ, Shiba E, Kobayashi T, Yayoi E, Furukawa J, Takatsuka Y, Shin E, Koyama H, Inaji H, Takai S: Prognostic impact of urokinase-type plasminogen activator (PA), PA inhibitor type-1 and tissue-type PA antigen levels in node-negative breast cancer: a prospective study on multicenter basis. Clin Cancer Res 1998;4:177-182.

7 Foekens JA, Peters HA, Look MP, Portengen H, Schmitt M, Kramer MD, Brünner N, Jänicke F, Meijer-van Gelder ME, Henzen-Logmans SC, van Putten WL, Klijn JG: The urokinase system of plasminogen activation and prognosis in 2780 breast cancer patients. Cancer Res 2000;60:636-643.
8 Cufer T, Borstnar S, Vrhovec I: Prognostic and predictive value of the urokinase-type plasminogen activator (uPA) and its inhibitors PAI-1 and PAI-2 in operable breast cancer. Int J Biol Markers 2003;18:106-115.

9 Harbeck N, Kates RE, Gauger K, Willems A, Kiechle M, Magdolen V, Schmitt M: Urokinasetype plasminogen activator uPA and its inhibitor PAI-1: novel tumour-derived factors with a high prognostic and predictive impact in breast cancer. Thromb Haemost 2004;91:450-456.

10 Harbeck N, Kates RE, Schmitt M: Clinical relevance of invasion factors urokinase-type plasminogen activator and plasminogen activator inhibitor type 1 for individualised therapy decisions in primary breast cancer is greatest when used in combination. J Clin Oncol 2002;20:1000-1007. 
11 Konecny G, Untch M, Arboleda J, et al.: Her2/neu and urokinase-type plasminogen activator and its inhibitor in breast cancer. Clin Cancer Res 2001; 7:2448-2457.

12 Bouchet C, Ferrero-Pous M, Hacene K, Becette V, Spyratos F: Limited prognostic value of c-erbB-2 compared to UPA and PAI-1 in primary breast carcinoma. Int J Biol Markers 2003;18:207-217.

13 Zemzoum I, Kates RE, Ross JS, Dettmar P, Dutta M, Henrichs C, Yurdseven S, Höfler H, Kiechle M Schmitt M, Harbeck N: Invasion factors uPA PAI-1 and HER2 status provide independent and complementary information on patient outcome in node-negative breast cancer. J Clin Oncol 2003; 21:1022-1028.

14 Harbeck N, Kates RE, Look MP, Meijer-Van Gelder ME, Klijn JG, Krüger A, Kiechle M Jänicke F, Schmitt M, Foekens JA: Enhanced benefit from adjuvant chemotherapy in breast cancer patients classified high-risk according to urokinase-type plasminogen activator (uPA) and plasminogen activator inhibitor type $1(\mathrm{n}=3424)$. Cancer Res 2002;62:4617-4622.

15 Look MP, van Putten WLK, Duffy MJ, et al.: Pooled analysis of prognostic impact of urokinasetype plasminogen activator and its inhibitor PAI-1 in 8377 breast cancer patients. J Natl Cancer Inst 2002;94:116-128.
16 Harbeck N, Kates RE, Look MP, Foekens JA, on behalf of the pooled analysis study of the EORTC Receptor and Biomarker Group (RBG): Pooled analysis $(n=8,377)$ evaluates predictive impact of uPA and PAI-1 for response to adjuvant therapy in breast cancer. J Clin Oncol 2004;23:523.

17 Hayes DF, Bast R, Desch CE, Fritsche H Jr, Kemeny NE, Jessup JM, Locker GY, Macdonald JS, Mennel RG, Norton L, Ravdin P, Taube S, Winn RJ: A tumour marker utility grading system (TMUGS): a framework to evaluate clinical utility of tumour markers. J Natl Cancer Inst 1996;88: 1456-1466.

18 Jänicke F, Prechtl A, Thomssen C, Harbeck N, Meisner C, Untch M, Sweep CG, Selbmann HK, Graeff H, Schmitt M; German N0 Study Group: Randomised adjuvant chemotherapy trial in highrisk, lymph node-negative breast cancer patients identified by urokinase-type plasminogen activator and plasminogen activator inhibitor type 1 . J Natl Cancer Inst 2001;93:913-920.

19 Harbeck N, Meisner C, Prechtl A, et al.: Level-I evidence for prognostic and predictive impact of uPA and PAI-1 in node-negative breast cancer provided by second scheduled analysis of multicenter Chemo-N0 therapy trial. Breast Cancer Res Treat 2001;69:213.

20 Euler U: Comparison of outcome prediction in node-negative breast cancer based on biomarkers uPA/PAI-1 or Adjuvant Online using the 10-year follow-up of the randomised multicenter Chemo N0 trial. ASCO Annual Meeting 2006; abstr 534.
1 Goldhirsch A, Glick JH, Gelber RD, Coates AS, Thürlimann B, Senn HJ; Panel members: Meeting highlights: international expert consensus on the primary therapy of early breast cancer 2005 . Ann Oncol 2005;16:1569-1583.

22 Roché H, Fumoleau P, Spielmann M, et al.: Sequential adjuvant epirubicin based and docetaxel chemotherapy for node-positive breast cancer patients: The FNCLCC PACS 01 Trial. J Clin Oncol 2006;24:5664-5671.

23 AGO 2008 guidelines for treatment of early and advanced breast cancer: www.ago-online.de.

24 Sweep CG, Geurts-Moespot J, Grebenschikov N, et al.: External quality assessment of trans-European multicentre antigen determination (enzymelinked immunosorbent assay) of urokinase-type plasminogen activator (uPA) and its type 1 inhibitor (PAI-1) in human breast cancer tissue extracts. Br J Cancer 1998;78:1434-1441.

25 Sweep FC, Fritsche HA, Gion M, Klee GG, Schmitt M; EORTC-NCI Working Group: Considerations on development, validation, application and quality control of immuno(metric) biomarker assays in clinical cancer research: an EORTC-NCI working group report. Int J Oncol 2003;23:17151726.

26 Harris L, Fritsche H, Mennel R, Norton L, Ravdin P, Taube S, Somerfield MR, Bast RC Jr; American Society of Clinical Oncology: American Society of Clinical Oncology 2007 update of recommendations for the use of tumour markers in breast cancer. J Clin Oncol 2007;25:5287-5312. 